\title{
New Active Damping Strategy for LCL-Filter-Based Grid-Connected Inverters with Harmonics Compensation
}

\author{
Guozhen $\mathrm{Hu}^{\dagger}$, Changsong Chen*, and Duan Shanxu* \\ 'Dept. of Electronic and Electrical Eng., WuHan Textile University, Wuhan,China \\ *Dept. of Electrical and Electronic Eng., Huazhong University of Science\& Technology, Wuhan, China
}

\begin{abstract}
The use of LCL filters in pulse width modulation voltage source converters is a standard solution for providing proper attenuation of high-order grid-current harmonics. However, these filters can cause the undesired effect of resonance. This paper proposes an active damping strategy with harmonics compensation. It can alleviate the harmonics around the resonance frequency caused by the LCL filters. The proposed strategy is attractive since it is simple, does not depend on grid parameters and does not increase the number of sensors. Simulation and experimental results verify the effectiveness of the proposed active damping strategy.
\end{abstract}

Key words: Active damping, Grid-connected inverter, Harmonics, LCL

\section{INTRODUCTION}

Distributed Generation (DG) systems based on renewable energy sources are experiencing rapid development. Pulse width modulation (PWM) voltage source inverters (VSI) are increasingly used to connect these different sources to power system [1], [2].

For the reduction of grid current ripples, LCL filters are often used as the output stage of grid-connected inverters. Nevertheless, LCL filters bring undesired resonance effects, which are caused by the zero impedance for some higher order harmonics of grid-connected current [3], [4].

In order to solve this problem, passive and active damping methods have been presented in some literatures. Passive damping is a simple way to guarantee system stability, but there is a decrease in the overall system efficiency caused by the added resistance [5]. To avoid such losses, a method which is called "virtual resistor" was presented. However, it requires an extra capacitor current sensor [6],[7]. Furthermore, two or even three loop active damping control is adopted. A method proposed by Blasko

Manuscript received May 7, 2012; revised Jan. 26, 2013

Recommended for publication by Associate Editor Se-Kyo Chung.

${ }^{\dagger}$ Corresponding Author:gzhhust@gmail.com

Tel: +86-13545161660, WuHan Textile University

*Dept. of Electrical and Electronic Eng., Huazhong University of

Science \& Technology, China and Kaura based on a lead-lag element can alleviate the effects of LCL resonance [8]. In [9] a grid side current with addition feedback of the current of the filter capacitor is shown. In addition, the methods proposed by Fei Liu et.al. in [10] and Guoqiao Shen et.al. in [11] realize the purposes of active damping through measuring two inductance currents. All of the above approaches require the measurement of extra capacitor voltage or current to achieve active damping.

The additional sensors increase the costs and complexity of the system design. So some resonance damping methods based on higher order controllers have been discussed. The method proposed by Liserre et al. does not apply additional sensors, but a genetic algorithm is used to tune the system, which complicates the final control algorithm [12]. Other methods to modify the current controller structure based on the use of digital filters are reviewed in [13]. However, these methods are sensitive to parameter uncertainties.

This paper proposes a simple active damping control strategy, which adopts harmonics compensation control. The proposed strategy has the following features: simple implementation, avoidance of additional costs, high reliability and freedom from dependence on grid parameters. In Section II the modeling and control of a grid-connected system are presented based on an LCL filter. In Section III the proposed active damping strategy based on harmonic compensation is introduced in detail. Section IV and Section V presented the simulation and experimental results. 


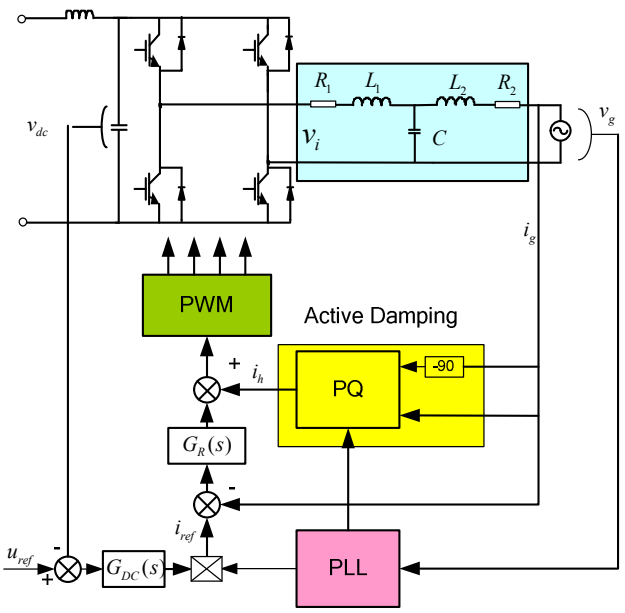

Fig. 1. Proposed LCL filter system of single-phase grid-connected. Inverter.

TABLE I

SYSTEM PARAMETERS

\begin{tabular}{|c|c|c|}
\hline Symbol & Quantity & Value \\
\hline$L_{l}$ & Converter-side filter inductance & $0.35 \mathrm{mH}$ \\
\hline$C$ & Filter capacitance & $5 \mathrm{uF}$ \\
\hline$L_{2}$ & Grid-side filter inductance & $0.35 \mathrm{mH}$ \\
\hline$R 1, R 2$ & Equivalent series resistors of $\mathrm{L}_{1}, \mathrm{~L}_{2}$ & $0.03 \Omega$ \\
\hline$R d$ & Equivalent series resistor of $\mathrm{C}$ & $0.1 \Omega$ \\
\hline$V_{d c}$ & Voltage of DC-link & $390 \mathrm{~V}$ \\
\hline$v_{g}$ & Voltage of grid & $220 \mathrm{VAC}$ \\
\hline$f_{0,}, f_{S, f c}$ & Grid frequency, Switch Frequency, & $50 \mathrm{~Hz}, 12.5 \mathrm{kHz}$, \\
& Control Frequency & $25 \mathrm{kHz}$ \\
\hline$L g$ & Grid inductance & $0.1 \mathrm{mH}$ \\
\hline
\end{tabular}

\section{SYSTEM MODELING AND CONTROL}

Fig. 1 shows the system topology and overall control structure of a $5-\mathrm{kW}$ grid-connected VSI inverter. The topology has an LCL filter connecting the inverter output with the grid. $L_{1}$ is the inverter-side inductance; $L_{2}$ is the grid-side inductance; and $R_{1}$ and $R_{2}$ are their equivalent series resistors, respectively. $C$ is the capacitance of the LCL filter and $R_{d}$ is the equivalent series resistor of $C$, which may be used for the purpose of resonance damping. $L_{g}$ is the grid inductance. The related parameters are given in Table I.

\section{A. Modeling}

Based on the system configuration of the LCL filter, the

$$
\begin{gathered}
G_{1}(s)=\frac{I_{L 1}(s)}{V_{i}(s)}=\frac{z_{22}}{z_{12} z_{21}-z_{11} z_{22}}=\frac{L_{2} C s^{2}+\left(R_{d}+R_{2}\right) C s+1}{s^{3} L_{1} L_{2} C+\left(L_{1} C\left(R_{2}+R_{d}\right)+L_{2} C\left(R_{1}+R_{d}\right)\right) s^{2}+\left(L_{1}+L_{2}+R_{1} C\left(R_{2}+R_{d}\right)+R_{2} R_{d} C\right) s+R_{1}+R_{2}} \\
G_{2}(s)=\frac{I_{g}(s)}{V_{i}(s)}=\frac{z_{12}}{z_{12} z_{21}-z_{11} z_{22}}=\frac{R_{d} C s+1}{s^{3} L_{1} L_{2} C+\left(L_{1} C\left(R_{2}+R_{d}\right)+L_{2} C\left(R_{1}+R_{d}\right)\right) s^{2}+\left(L_{1}+L_{2}+R_{1} C\left(R_{2}+R_{d}\right)+R_{2} R_{d} C\right) s+R_{1}+R_{2}}
\end{gathered}
$$




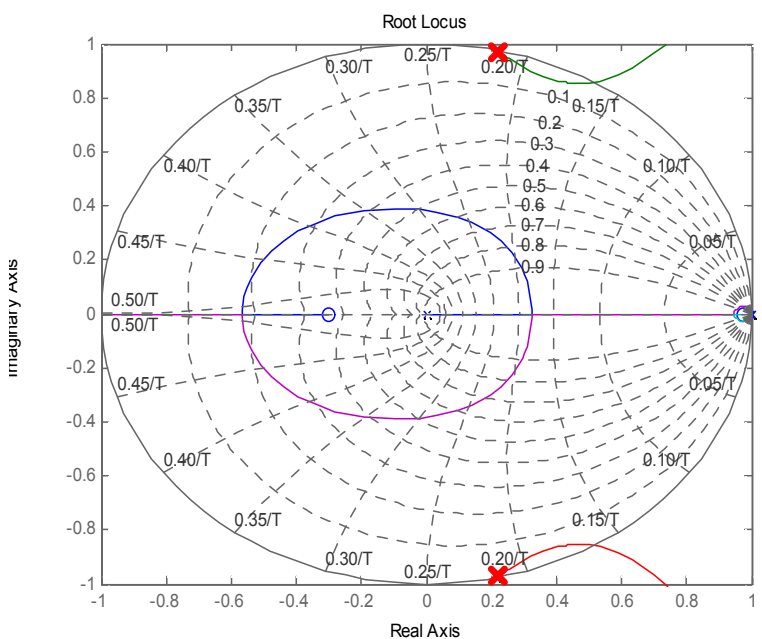

(a) Grid side current control. Root Locus

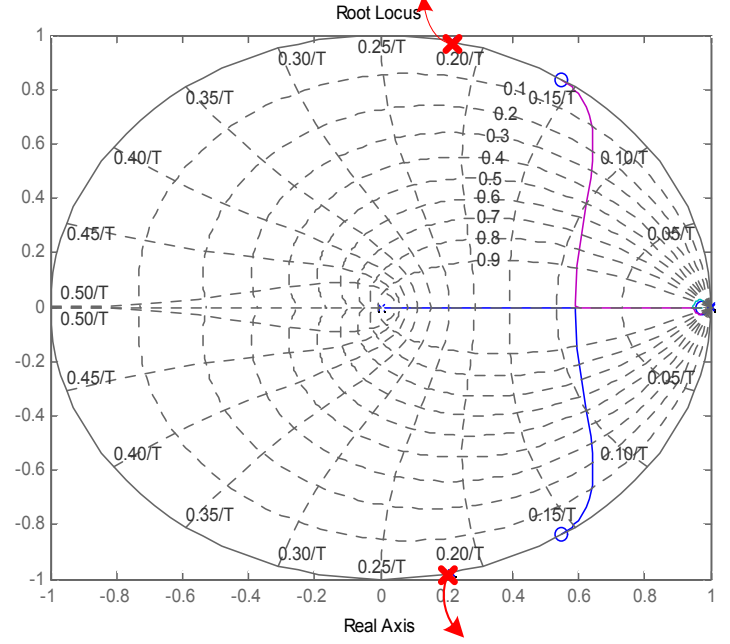

(b) Converter side current control.

Fig. 2. Root locus of these two control methods.

$$
G_{R}(s)=K_{p}+\sum_{n=1,3,5 \ldots} \frac{2 \omega_{c} K_{r n} s}{s^{2}+2 \omega_{c} s+\left(n \omega_{0}\right)^{2}}
$$

Where $K_{p}$ is the proportional gain; $K_{r n}$ is the resonance gain of the PR controller; $\omega_{0}$ is fundamental frequency; and $\omega_{c}$ is the bandwidth of the resonant compensator to reduce the sensitivity toward frequency variations of the grid.

The difference between an LCL filter and a single L filter mainly reflected the attenuation of the high-frequency harmonics. In addition, these two filers have the same characteristics in the low-frequency region. As a result, a controller with an LCL filter can be designed according to a single L filter. Reference [15] gives the classic PI and PR controller design method. The parameter values can be calculated as follows:

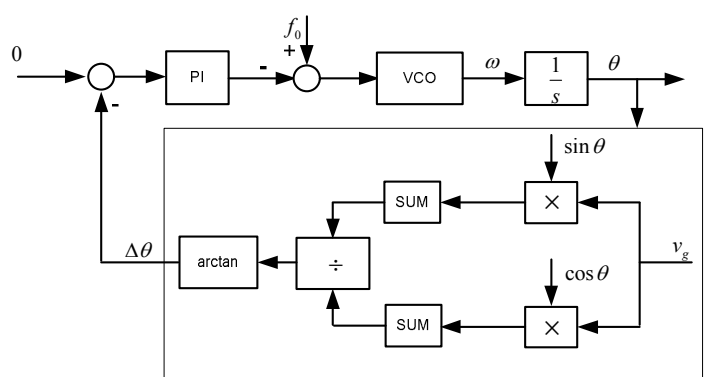

Fig. 3. Realization of DFT PLL method.

$$
\left\{\begin{array}{l}
K_{P}=\frac{L_{T}}{2 T_{s y s}}=\frac{L_{1}+L_{2}}{2 T_{s y s}} \\
K_{I}=\frac{L_{T}}{R}=\frac{L_{1}+L_{2}}{R_{1}+R_{2}}=\delta K_{r}
\end{array}\right.
$$

Where $T_{s y s}$ is the sampling frequency and $\delta$ is the coefficient (2 3).

Although a zero crossing detection PLL could be implemented easily and conveniently, they are unable to function properly when the grid voltage has multi-zero-crossings resulting from harmonics or spikes. To overcome this problem, a phase detection method based on the Discrete Fourier Transformation (DFT) is adopted in this paper [16]. The DFT phase detection method can separate the frequency, phase and magnitude of the fundamental frequency signal. The realization of the DFT is shown in Fig.3. $\theta$ is the phase angle of the grid voltage and $\Delta \theta$ is the phase error.

\section{PROPOSED ACTIVE DAMPING BASED ON HARMONICS COMPENSATION}

Ideally, the spectrum of the grid-connected current only consists of the fundamental component and the switching frequency harmonics. However, the resonance effect of the LCL filters would bring about high order harmonics. Therefore, the harmonics components of the LCL grid-connected current $i_{h}$ consist of three frequency components, as shown in Fig.4. Where $i_{\text {low }}$ is the low-order current harmonics, which is caused by some nonlinear factor, such as modulation or dead zone. $i_{s w}$ is the harmonics current of the switching frequency. And $i_{s w}$ is related to the filters parameters. $i_{\text {low }}$ can be restrained through proper controller design, such as the PR controller mentioned in Section II. $i_{\text {res }}$ is the harmonics around the resonance frequency and its magnitude is related to the performance of the active damping. The proposed method is used to compensate the harmonics $i_{\text {res }}$. 


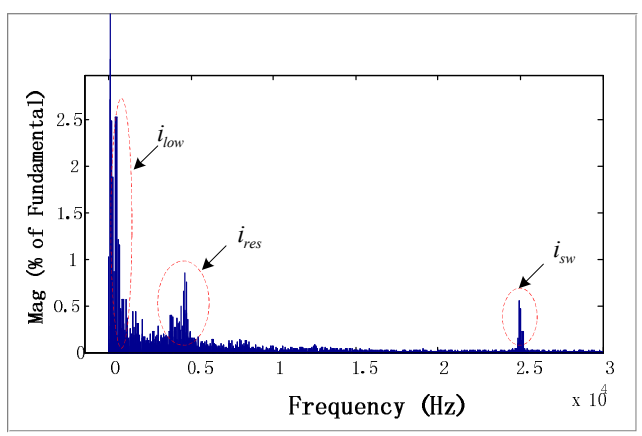

Fig. 4. Spectrum of grid-connected current.

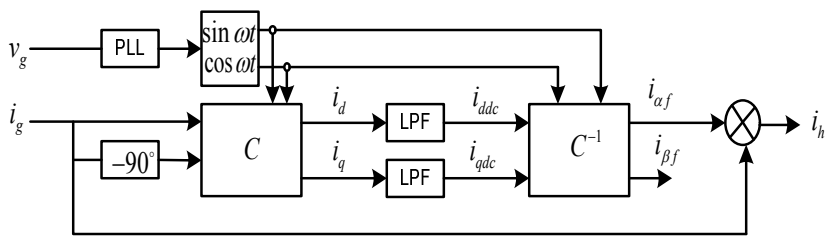

Fig. 5. Diagram of Harmonic detection.

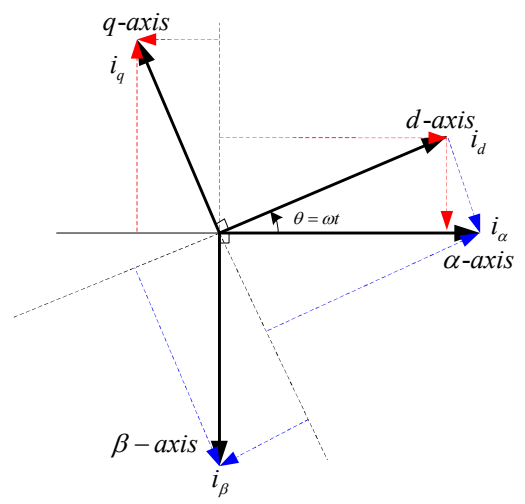

Fig. 6. Vector diagram for single-phase inverter.

\section{A. Active Damping Strategy}

A power theory method was adopted in this paper to compensate the ac component $i_{\text {res }}$ of the grid-connected current to reduce the effect caused by the LCL resonance. The extraction of the harmonic current is the key factor of the proposed active damping method. Usually, a variety of harmonic detection methods are used in active filters. Analog filters can be used to extract the harmonic component. However, they are complex and the device is susceptible to interference caused by the detection of distortion. The Fourier decomposition method requires a cycle of delay, and the computability and real-time are not good.

A harmonic detection method based on the instantaneous power theory is simple and its real time is good. This method is widely used in three-phase power systems. For single-phase grid-connected systems, an imaginary current must be constructed to form the two-phase stationary $\alpha-\beta$ coordinate system. In this paper, an imaginary current which lags the actual current $90^{\circ}$ is adopted, which can be written as:

$$
\left\{\begin{array}{l}
i_{\alpha}=i_{g}=\sum_{h=1,3,5 \ldots}^{\infty} I_{g h} \cos \left(h \omega t+\phi_{h}\right) \\
i_{\beta}=\sum_{h=1,3,5 \ldots}^{\infty} I_{g h} \cos \left(h\left(\omega t-90^{\circ}\right)+\phi_{h}\right)
\end{array}\right.
$$

Where $I_{g h}$ is the harmonic amplitude of $i_{g}$. A harmonic detection diagram for a single-phase grid-connected system is shown in Fig.5. Harmonic detection can be realized with the following steps.

Firstly, the phase current in the stationary $\alpha-\beta$ coordinates should be transformed to the synchronous $d-q$ rotating coordinates. The phase angle $\phi$ is derived from a PLL. A vector diagram of the $\alpha-\beta$ coordinates to the $d-q$ coordinates is shown in Fig.6. The current vector $\left[i_{\alpha}\right.$ $\left.i_{\beta}\right]$ in the $\alpha-\beta$ coordinates can be transformed in the $d-q$ coordinates as:

$$
\left[\begin{array}{l}
i_{d} \\
i_{q}
\end{array}\right]=C\left[\begin{array}{l}
i_{\alpha} \\
i_{\beta}
\end{array}\right]=\left[\begin{array}{c}
I_{g} \cos \phi \\
I_{g} \sin \phi
\end{array}\right]+\left[\begin{array}{l}
i_{d a c} \\
i_{q a c}
\end{array}\right]=\left[\begin{array}{l}
i_{d d c} \\
i_{q d c}
\end{array}\right]+\left[\begin{array}{c}
i_{d a c} \\
i_{q a c}
\end{array}\right]
$$

Where $C=\left[\begin{array}{cc}\cos \omega t & \sin \omega t \\ -\sin \omega t & \cos \omega t\end{array}\right] . i_{d}$ and $i_{q}$ consist of ac components ( $i_{d a c}$ and $i_{q a c}$, respectively) and dc components ( $i_{d d c}$ and $i_{q d c}$, respectively). $i_{d d c}$ and $i_{q d c}$ are obtained through a low pass filter (LPF). For a grid-connected system, $i_{q d c}$ is equal to zero.

Secondly, an inverse transformation is carried out to obtain the fundamental current emitted to the grid. $i_{\alpha f}$ and $i_{\beta f}$ can be achieved by (8).

$$
\left[\begin{array}{l}
i_{\alpha f} \\
i_{\beta f}
\end{array}\right]=C^{-1}\left[\begin{array}{l}
i_{d d c} \\
i_{q d c}
\end{array}\right]=\left[\begin{array}{l}
I_{g} \cos (\omega t+\phi) \\
I_{g} \cos \left(\omega t+\phi-90^{\circ}\right)
\end{array}\right]
$$

From (7) and (8), the compensated current harmonics of the proposed strategy can be calculated as:

$$
i_{h}=i_{g}-i_{\alpha f}
$$

As shown in Fig.1, the current harmonics $i_{h}$ is added to the output of the PR controller. So the harmonics coming from the output current can be compensated. The proposed compensation strategy is simple, clear, and easy to implement.

As shown in Fig.5, a suitable low pass filter should be used to achieve the dc components $i_{d d c}$ and $i_{q d c}$. In addition, its order and phase delay are considered to be as low as possible. An iterative optimization using the filter design and analysis tool from Matlab was carried out. The IIR Butterworth LPF was found to meet the specifications. The description of the IIR can be written as: 


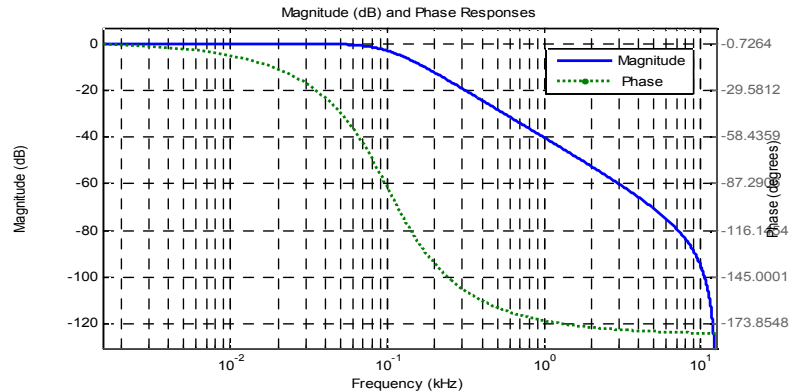

(a) Frequency characteristic of designed IIR filter.

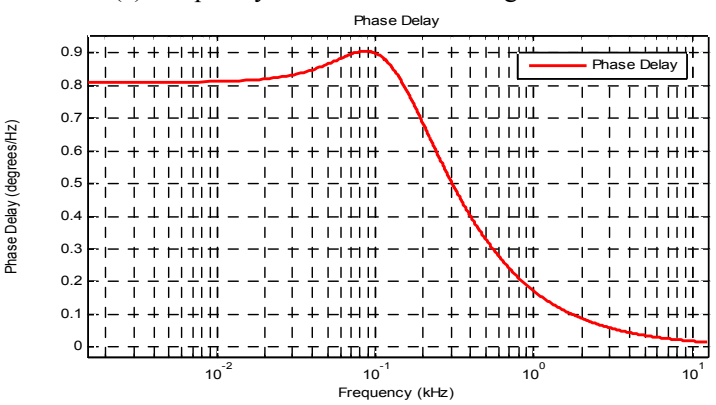

(b) Phase delay of designed IIR filter.

Fig. 7. Bode plot of IIR Butterworth Low Pass Filter.

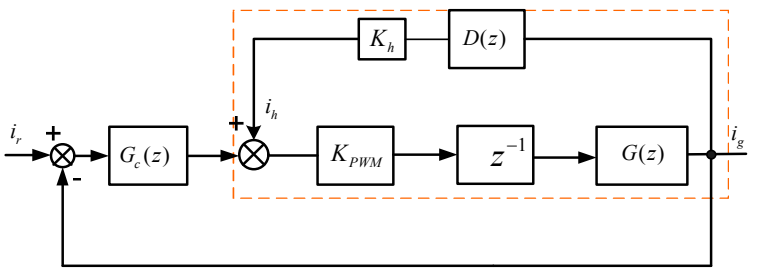

Fig. 8. Block Diagram of the Proposed Active Damping Method.

\section{B. Analysis of the Damping Performance}

$$
H(z)=\frac{Y(z)}{X(z)}=\frac{\sum_{i=0}^{M} a_{i} z^{-i}}{1-\sum_{i=1}^{N} b_{i} z^{-i}}
$$

With regard to the specifications, the IIR controller was designed as:

$$
H(z)=\frac{1.55148 e-4+(3.10296 e-4) z^{-1}+(1.55148 e-4) z^{-2}}{1-1.9645 z^{-1}+0.96508 z^{-2}}
$$

The filter cutoff frequency is designed to be $100 \mathrm{~Hz}$. Fig. 7 shows a characteristic Bode plot of the designed IIR filter.

The Proposed Active Damping strategy can be shown as Fig.8. The Active Damping block using the power theory can be equivalent to $D(z) . K_{h}$ is coefficient of the compensation, which can be used to adjust the amplitude of the compensation to get a better damping effect. $D(z)$ can be shown as (12).

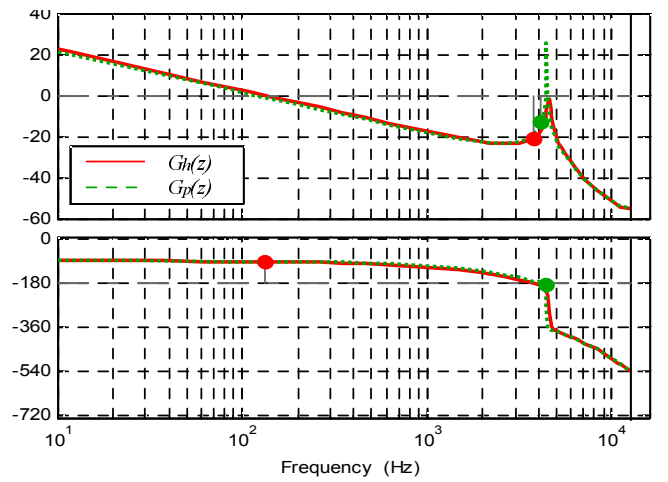

Fig. 9. Bode diagrams of $G_{p}(z)$ and $G_{h}(z)$.

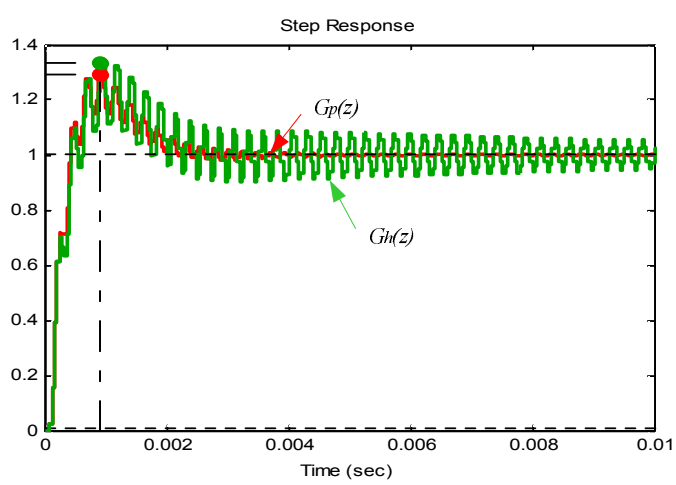

Fig. 10. Step response of close loop control system.

$$
D(z)=1-H(z)=\frac{1-\left(\sum_{i=0}^{M} a_{i} z^{-i}+\sum_{i=0}^{N} b_{i} z^{-i}\right)}{1-\sum_{i=1}^{N} b_{i} z^{-i}}
$$

Without harmonics compensation, the discrete time-domain open-loop transfer function $G_{p}(z)$ can be obtained by converting $G 2(s)$ from the continuous to discrete time form $G(z)$ :

$$
G_{p}(z)=K_{p w m} z^{-1} Z\left(\frac{1-e^{-s T_{s s s}}}{s} G_{2}(s)\right)=K_{p w m} z^{-1} G(z)
$$

In addition, with the proposed strategy, the discrete time-domain open-loop transfer function can be obtained as $G_{h}(z)$ :

$$
G_{h}(z)=\frac{K_{p w m} z^{-1} G_{2}(z)}{1-D(z) K_{h} K_{p w m} z^{-1} G_{2}(z)}
$$

Fig. 9 shows a Bode diagrams of $G_{p}(z)$ and $G_{h}(z)$. The resonant peak of $G_{h}(z)$ is lower than that of $G_{p}(z)$, which is below $0 \mathrm{~dB}$. The magnitude margin of $G_{h}(z)$ is $20 \mathrm{~dB}$, which is also higher than that of $G_{p}(z)$.

Fig. 10 shows the step response of the close loop control system. The proposed strategy has better dynamic performance. In addition, it can be seen from Fig. 9 that the system experiences resonance without harmonics compensation. 


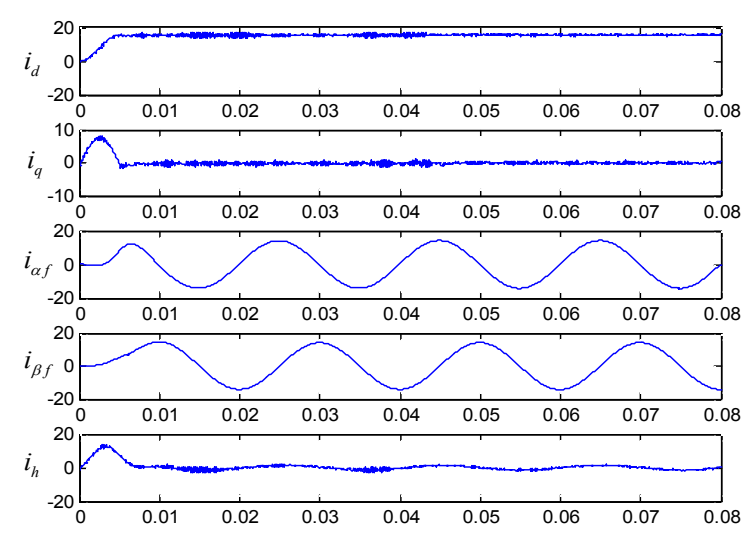

Fig. 11. Simulation results of $d-q, \alpha-\beta$ coordinates and harmonic current.
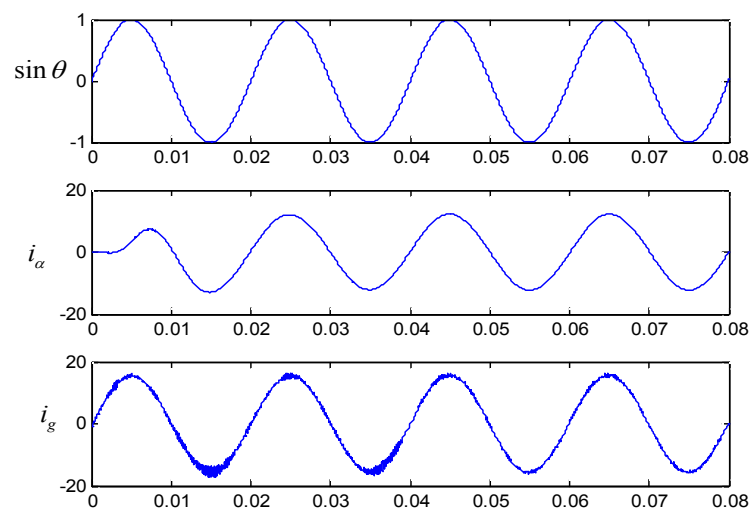

Fig. 12. Impact of Phase delay of IIR filter.

\section{SiMULATION RESULTS}

To confirm the validity of the proposed active damping strategy, simulations have been performed using Matlab/Simulink. The simulation studies were operated under the parameters given in Table I.

Fig.11 shows the simulated waveforms of $i_{d}$ and $i_{q}$ (the current of the $d-q$ coordinates) as well as $i_{\alpha f}$ and $i_{\beta f}$ (the current of the $\alpha-\beta$ coordinates through the $d q-\alpha \beta$ transformation). $i_{d}$ is a purely de value and $i_{q}$ is equal to zero. $i_{h}$ is the compensate harmonics extracted from the output current.

As can be seen from Fig.12, the phase delay of the designed IIR filter has less impact on the system.

Fig. 13 shows the simulated waveforms of the output currents when the grid voltage has no distortion. The proposed strategy is activated at $0.04 \mathrm{~s}$. The resonance is clearly visible in the grid-connected current before $0.04 \mathrm{~s}$. In addition, the harmonic current compensation as an active damping block can correctly attenuate the existing oscillations after $0.04 \mathrm{~s}$. This can also be seen from the current spectrum as Fig.13 (b) and Fig.13(c). The magnitude of the output current around the resonance frequency has been alleviated through the proposed active damping strategy.

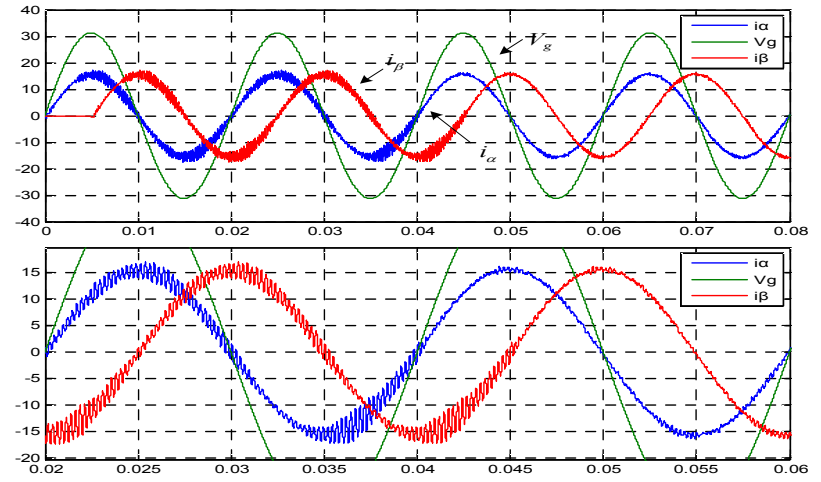

(a) Grid-connected current $\left(i_{\alpha}\right)$, imaginary current $\left(i_{\beta}\right)$, Ideal Grid

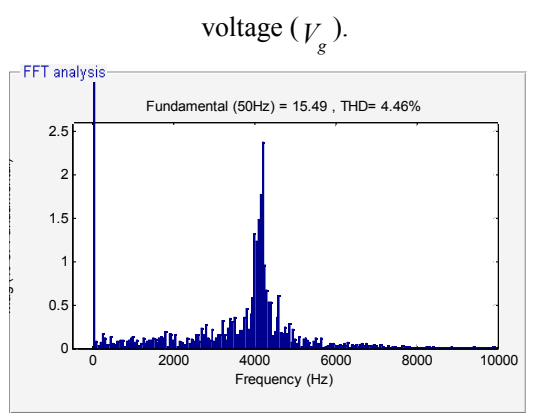

(b) Spectrum analysis of output current without compensation.

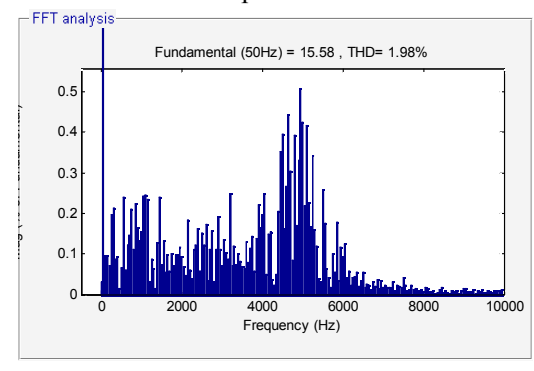

(c) Spectrum analysis of output current with compensation.

Fig. 13. Simulation results with the proposed control strategy activated at $0.04 \mathrm{~s}$.

Because the harmonics of the output current are extracted with the power theory (Section IV), the proposed strategy can be immune to grid voltage background harmonics. As shown in Fig.14 (a), the distortion of grid voltage (VTHD $=4.65 \%$ ) does not affect the effectiveness of the proposed strategy. Furthermore, the resonance of the LCL filters is strongly related to the grid impedance. The resonant frequency and magnitude vary with changes of the grid impedance. As shown in Fig.14 (b), the proposed strategy can also be effective when the grid inductance $\mathrm{Lg}=1 \mathrm{mH}$.

\section{EXPERIMENTAL RESULTS}

As shown in Fig.15, a 5-kW single-phase LCL grid-connected prototype is built to verify the proposed control strategy. The parameters are the same as those in the foregoing simulation. 


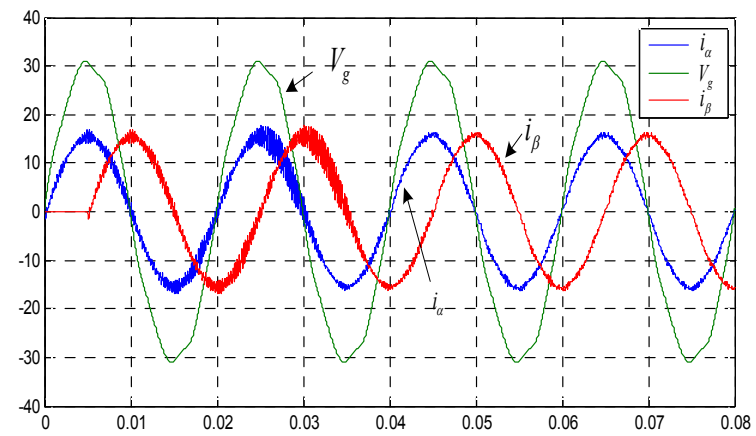

(a) Validity of the proposed strategy when $\mathrm{V}_{\mathrm{THD}}=4.65 \%$.

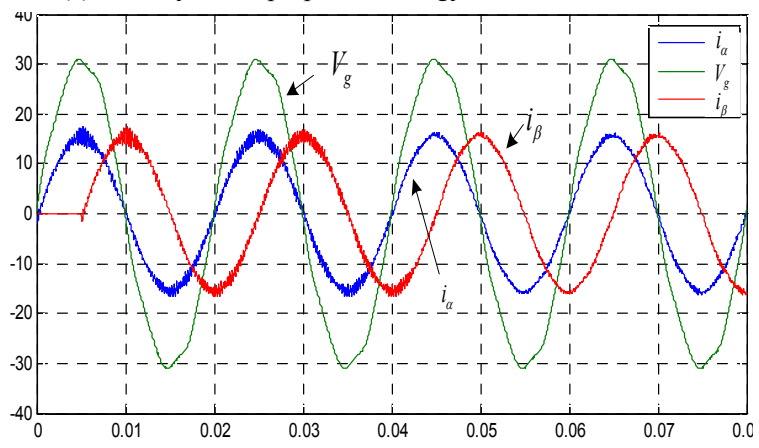

(b) Validity of the proposed strategy when $L g=1 \mathrm{mH}$.

Fig. 14. Simulation results of the proposed control affected by distortion of grid voltage.

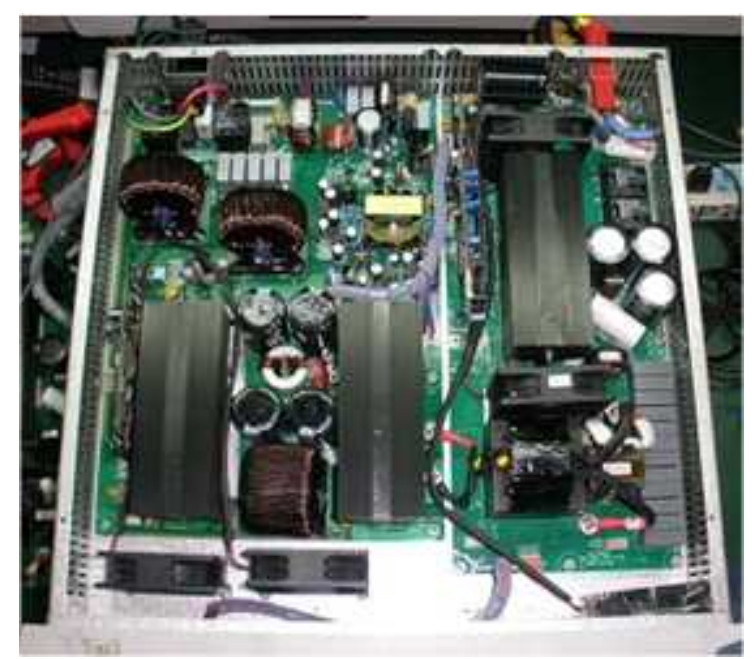

Fig. 15. 5-kW single-phase LCL grid-connected prototype.

The grid voltage and its spectrum are shown in Fig.16. Its total harmonic distortion (THD) is $2.74 \%$.

The effectiveness of the proposed strategy can be verified by Fig.17 (a) and Fig.17 (b). The current harmonics caused by resonance are effectively eliminated by the proposed active damming strategy. Fig.17(c) shows the results of the harmonics around the resonant frequency before and after compensation.

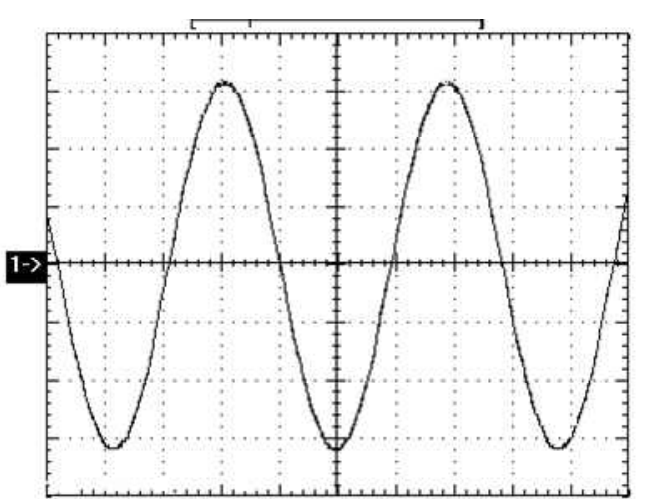

(a) Grid voltage.

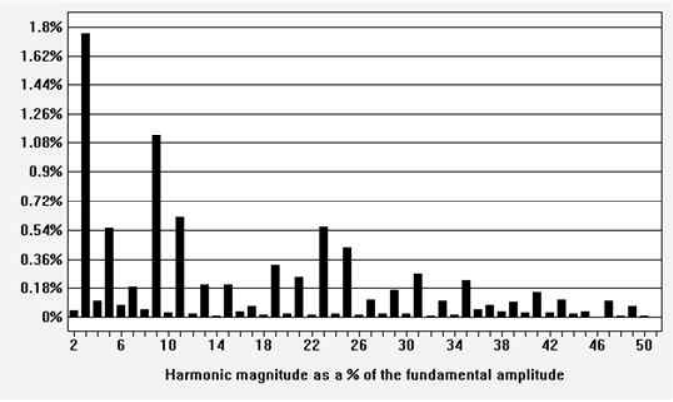

(b) Spectrum of grid voltage.

Fig. 16. Waveform of Grid voltage and its THD is $2.74 \%$.

The effectiveness of the proposed strategy can be verified by Fig.17 (a) and Fig.17 (b). The current harmonics caused by resonance is effectively eliminated by the proposed active damming strategy. Fig.17(c) shows the results of the harmonics around resonant frequency before and after compensation.

The THD of the grid-connected current is increased to $3.25 \%$ as shown in Fig. 17(d).

\section{CONCLUSIONS}

This paper has presented a simple active damping solution to improve the system performance. And the current harmonics caused by resonance is compensated by the proposed strategy. Simulations and experiments have been performed with a $5 \mathrm{~kW}$ experimental setup to verify the effectiveness. The strategy does not increase additional sensors and is easy to implement, which is suitable for industrial applications.

\section{ACKNOWLEDGMENT}

The authors would like to thank financial supports provided by National Basic Research Program of China (973 Program) and its project number is 2010CB227206. 


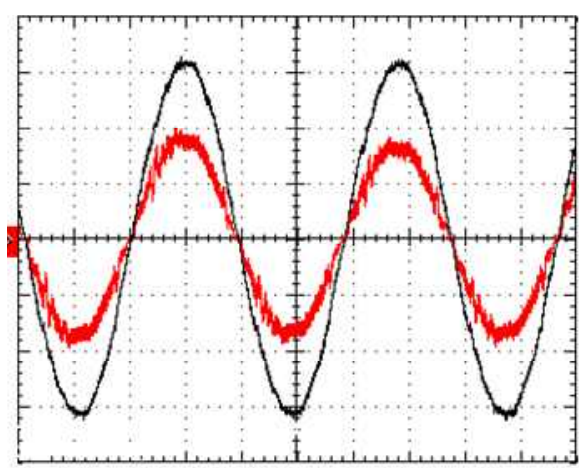

(a) Experimental result of current without compensation.

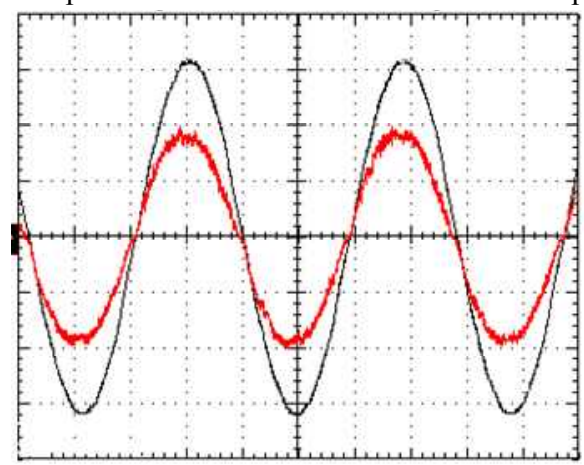

(b) Experimental result of current with proposed strategy.

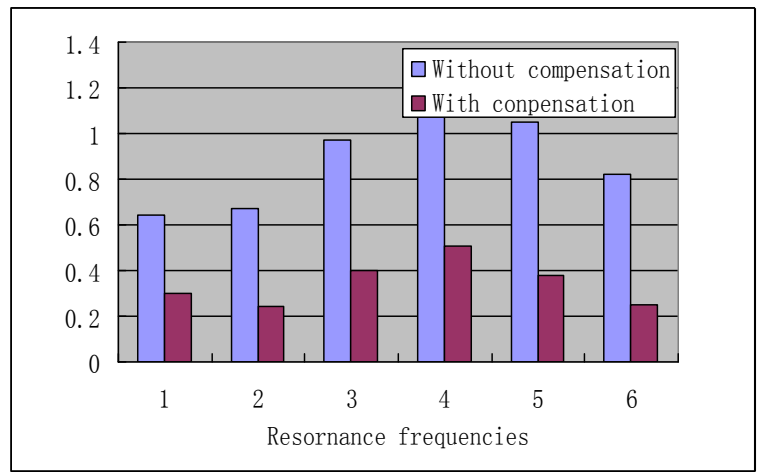

(c) Comparison of harmonics around resonant frequency.

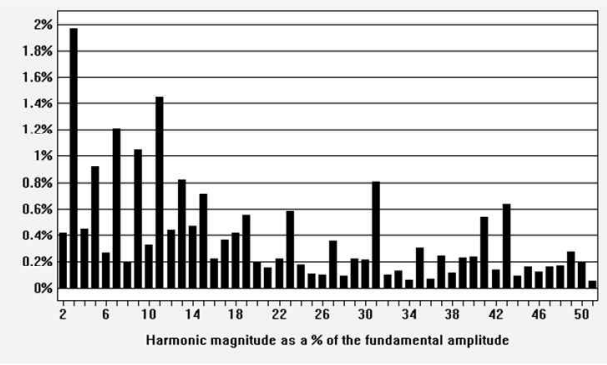

(d) THD of grid-connected current with proposed strategy (THD $=3.25 \%)$.

Fig. 17. Experimental results with proposed strategy (Ch1: Grid voltage, 100V/div, Ch2: Grid-side current, 5A/div).

\section{REFERENCES}

[1] R.-J. Wai and W.-H. Wang, "Grid-connected photovoltaic generation system," IEEE Trans. Circuits and Syst., Vol.
55,No. 3, pp. 953-964, Apr. 2008.

[2] J. L. Agorreta, M. Borrega, and J. Lopez, "Modeling and control of n-paralleled grid-connected inverters with lcl filter coupled due to grid impedance in pv plants," IEEE Trans. Power Electron., Vol. 26, No. 3, pp. 770-785, Mar. 2011.

[3] S. Cobreces, E. Bueno, F. J. Rodriguez, F. Huerta, and P. Rodriguez, "Influence analysis of the effects of an inductive-resistive weak grid over L and LCL filter current hysteresis controllers," in European Conference on Power Electronics and Applications, pp. 1-10, Sep. 2007.

[4] S.-Y. Park, C.-L. Chen, and J.-S. Lai, "Admittance compensation in current loop control for a grid-tie lcl fuel cell inverter," IEEE Trans. Power Electron., Vol. 23,No. 4,pp. 1716-1723, Jul. 2008.

[5] M. Castilla, J. Miret, J. Matas, L. Garcia de Vicuna, J. M. Guerrero, "Control design guidelines for single-phase grid-connected photovoltaic inverters with damped resonant harmonic compensators," IEEE Trans. Ind. Electron., Vol. 56, No. 11, pp. 4492-4501, Nov. 2009.

[6] C. Wessels, J. Dannehl, and F. W. Fuchs, "Active damping of LCL-filter resonance based on virtual resistor for PWM rectifiers-stability analysis with different filter parameters," in Power Electronics Specialists Conference (PESC), pp.3532-3538, 2008.

[7] W. Gullvik, L. Norum, and R. Nilsen, "Active damping of resonance oscillations in LCL-filters based on virtual flux and virtual resistor," in European Conference on Power Electronics and Applications, pp.1-10, Sep. 2007.

[8] V. Blasko and V. Kaura, "A novel control to actively damp resonance in input lc filter of a three-phase," IEEE Trans. Ind. Appl., Vol. 33,No. 2,pp. 542-550, Mar./Apr. 1997.

[9] E. Twining and D. G. Holmes, "Grid current regulation of a three-phase voltage source inverter with an LCL input filter,"IEEE Trans. Power Electron., Vol. 18, No. 3, pp. 888-895, May 2003.

[10] F. Liu, Y. Zhou, S. Duan, J. Yin, B. Liu, and F. Liu, "Parameter design of a two-current-loop controller used in a grid-connected inverter system with lcl filter," IEEE Trans. Ind. Electron., Vol. 56, No. 11, pp. 4483-4491, Nov. 2009.

[11] G. Shen, X. Zhu, J. Zhang, and D. Xu, "A new feedback method for pr current control of lcl filter-based grid-connected inverter," IEEE Trans. Ind. Eletron., Vol. 57, No. 6, pp. 2033-2041,Jun. 2010.

[12] M. Liserre, A. Dell'Aquila, and F.Blaabjerg, "Genetic algorithm-based design of the active damping for an LCL-filter three-phase active rectifier," IEEE Trans. Power Electon., Vol. 19, No. 1, pp. 76-86, Jan. 2004.

[13] J. Dannehl, M. Liserre, and F. W. Fuchs, "Filter-based active damping of voltage source converters with lcl filter," IEEE Trans. Ind. Electron., Vol. 58, No. 8, pp. 3623-3633, Aug. 2011.

[14] F. Wang, J. L. Duarte, M. A. M. Hendrix, and P. F. Ribeiro. "Modeling and analysis of grid harmonic distortion impact of aggregated dg inverters," IEEE Trans. Power Electron. Vol. 26, No. 3, pp. 786-797, Mar. 2011.

[15] M. H. Bierhoff and F. W. Fuchs, "Active damping for three-phase pwm rectifiers with high-order line-side filters," IEEE Trans. Ind. Electron., Vol. 56, No. 2, pp. 371-379, Feb. 2009.

[16] K. Xue-juan, L. Fang, P. Li , and K. Yong. "Implement and parameter design of dpll for invertors based on cycle control," Proceeding of CSEE, Vol. 27, No. 1, pp. 1-10, 2007. 


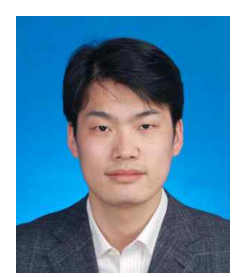

Guozhen Hu was born in Hubei Province, China, in 1979. He received his M.S. from the Chongqing University of Post and Telecommunications, Chongqing, China, in 2005. Since 2008, he has been working towards his Ph.D. at the Huazhong University of Science and Technology, Wuhan, China. His current research interests include the modeling and control of power converter systems, energy management and conversion techniques.

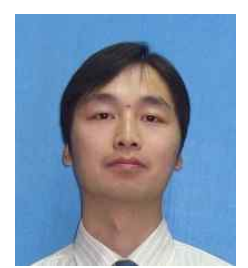

Changsong Chen was born in Hubei Province, China, in 1977. He received his M.S. and Ph.D. in Electrical Engineering from the Huazhong University of Science and Technology, Wuhan, China, in 2007 and 2011, respectively. His current research interests include power forecasting and energy management of renewable energy

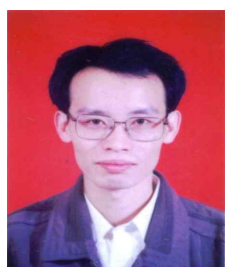

Duan Shanxu received his B.Eng., M.Eng., and Ph.D. in Electrical Engineering from the Huazhong University of Science and Technology, Wuhan, China, in 1991, 1994, and 1999, respectively. Since 1991, he has been a Faculty Member in the College of Electrical and Electronics Engineering, Huazhong University of Science and Technology, where he is currently a Professor. His main research interests include stabilization, nonlinear control with application to power electronic circuits and systems, fully digitalized control techniques for power electronics apparatus and systems, and optimal control theory and corresponding application techniques for high-frequency pulsewidth-modulation power converters. Dr. Duan is a Senior Member of the Chinese Society of Electrical Engineering and a Council Member of the Chinese Power Electronics Society. He was chosen as one of the New Century Excellent Talents by the Ministry of Education of China, in 2007, and he received the honor of "Delta Scholar" in 2009.

resources. 